\title{
Electron-screening correction for the proton-proton reaction
}

\author{
John N. Bahcall* \\ School of Natural Sciences, Institute for Advanced Study, Princeton, New Jersey 08540 \\ Xuelei Chen ${ }^{\dagger}$ and Marc Kamionkowski ${ }^{\ddagger}$ \\ Department of Physics, Columbia University, 538 West 120th Street, New York, New York 10027
}

(Received 23 December 1996; revised manuscript received 13 January 1998)

\begin{abstract}
We test the Salpeter formalism for the electron screening of the solar proton-proton fusion reaction by solving numerically the relevant Schrödinger equation. We evaluate exactly the square of the overlap integral of the two-proton wave function and the deuteron wave function and compare with the usual analytic approximation. The usual WKB solution agrees with the numerical result to $O\left(10^{-4}\right)$. The WKB approximation should be even more precise for the other nuclear fusion reactions in the $p p$ chain and CNO cycles. [S0556-2813(98)01505-2]
\end{abstract}

PACS number(s): 26.65.+t, 25.40.-h, 96.60.Hv, 97.10.Cv

Because of the importance of calculating precise nuclear fusion rates at low energy in connection with increasingly precise solar-neutrino experiments, attention has recently been drawn to the question of electron screening in solar fusion reactions [1-4]. Other authors have investigated in detail the validity of the determination of the screening potential [4]. The purpose of the present paper is to address a discrete and separate issue, namely, whether the standard WKB analytic approximation is sufficiently accurate to determine the screening correction to fusion rates to a percent or better given a specified screening potential.

We test in this paper the robustness of the standard WKB analytic treatment due to Salpeter [1] by solving numerically the relevant Schrödinger equation, including a Debye-Huckel screening potential, for the fundamental proton-proton $(p p)$ reaction. The unscreened rate of this reaction can be calculated precisely [5] using standard weak-interaction theory, accurate laboratory data for the two-proton system, and refined deuteron wave functions in agreement with a variety of nuclear-physics measurements. Radiative corrections are also included in the most recent calculation [5].

For our purposes, it is sufficient to use the standard Debye-Huckel potential, which a number of authors have recently found to be an accurate description of the screening effect around fusing ions in the solar interior [4]. However, our results do not depend upon the specific form of the potential we use. For completeness, we also carry out similar calculations with a different potential suggested by Dzitko et al. [2]. We obtain, as expected, a similarly precise description of the solution in the presence of the Dzitko et al. potential as for the standard Debye-Huckel potential.

In the following, we rederive Salpeter's analytic result for the weak-screening limit using a kinetic-theory approach (rather than Salpeter's thermodynamic arguments). Then, we calculate the proton-proton wave function in both a screened and unscreened Coulomb potential by numerical solution of

\footnotetext{
*Electronic address: jnb@ias.edu

${ }^{\dagger}$ Electronic address: xuelei@phys.columbia.edu

${ }^{\ddagger}$ Electronic address: kamion@phys.columbia.edu
}

the Schrödinger equation. We then use these results to evaluate numerically the electron-screening correction to the proton-proton reaction for both the Debye-Huckel and the Dzitko et al. potential, thereby testing the validity of the standard WKB calculation. Finally, we also calculate a correction to Salpeter's result and find it to be negligibly small.

To begin, we rederive Salpeter's screening correction. To do so, we use kinetic theory to calculate reaction rates in both a screened and unscreened plasma. Although Salpeter's derivation was based on a thermodynamic argument, this alternative approach recovers the same results, and it will be useful for understanding the numerical work that follows. With our analytic approach, a very small correction to Salpeter's results is obtained and presented at the end of this paper.

The nuclear fusion rates in the solar interior are controlled primarily by Coulomb barriers. Therefore, the energy dependence of the fusion cross section is usually written as

$$
\sigma(E) \equiv S(E) \exp (-2 \pi \eta) / E
$$

where $S(E)$ is a function that varies smoothly in the absence of resonances, and $\eta=Z_{1} Z_{2} e^{2} / \hbar v$. Here, $Z_{1} e$ and $Z_{2} e$ are the charges of the two colliding nuclei, and $v$ is their relative velocity.

The controlling factor, $\exp (-2 \pi \eta)$, in Eq. (1) takes into account the probability for the nuclei to tunnel through the Coulomb barrier. It is obtained from the Coulomb potential $V(r)=Z_{1} Z_{2} e^{2} / r$ through the WKB approximation,

$$
\begin{aligned}
\Gamma(E) & =\exp \left(\frac{-2}{\hbar} \int_{0}^{r_{c}}\left[2 \mu\left(V_{\text {Coul }}(r)-E\right)\right]^{1 / 2} d r\right) \\
& =\exp \left(-2 Z_{1} Z_{2} \frac{e^{2}}{\hbar} \sqrt{\frac{2 \mu}{E}} \int_{0}^{1} \sqrt{\frac{1}{u}-1} d u\right) \\
& =e^{-2 \pi \eta}
\end{aligned}
$$


where $r_{c}$ is the classical turning point, defined by $V_{\text {Coul }}\left(r_{c}\right)$ $=E, E$ is the kinetic energy, and $\mu=m_{1} m_{2} /\left(m_{1}+m_{2}\right)$ is the reduced mass. Here, $m_{1}$ and $m_{2}$ are the masses of the reacting nuclei.

If the energies of the reacting nuclei have a MaxwellBoltzmann distribution at a temperature $T$, the thermallyaveraged cross section times relative velocity is [6]

$$
\langle\sigma v\rangle=\sqrt{\frac{8}{\pi \mu\left(k_{B} T\right)^{3}}} \int_{0}^{\infty} d E S(E) \exp \left(-2 \pi \eta-E / k_{B} T\right) .
$$

However, in the stellar interior each nucleus, even though completely ionized, attracts neighboring electrons and repels neighboring nuclei; thus, the potential between two colliding nuclei is no longer a pure Coulomb potential, but a screened potential $V_{\mathrm{sc}}(r)$. In the weak-screening case, the Coulomb interaction energy between a nucleus and its nearest few electrons and nuclei of the gas is small compared with the thermal energy $k_{B} T$. In this case, the surrounding electrons and ions are only slightly displaced, and we obtain a screened potential of the form [1]

$$
V_{\mathrm{sc}}(r)=\frac{Z_{1} Z_{2} e^{2}}{r} \exp \left(-r / r_{D}\right)
$$

were $r_{D}=\zeta^{-1 / 2}\left(k_{B} T A / e^{2}\right)^{1 / 2} a$ is the Debye radius for the cloud; $A=A_{1} A_{2} /\left(A_{1}+A_{2}\right)$ is the reduced mass in atomic mass units; $\zeta=\sqrt{\sum_{i}\left(X_{i} Z_{i}^{2} / A_{i}+X_{i} Z_{i} / A_{i}\right)} ; X_{i}, Z_{i}$, and $A_{i}$ are the mass fraction, charge, and mass number, respectively, of nucleus $i$;

$$
a=\frac{1}{\left(4 \pi \rho N_{0}\right)^{1 / 3}}=\rho^{-1 / 3}\left(0.51 \times 10^{-8} \mathrm{~cm}\right)
$$

is a measure of interparticle distance; $\rho$ is the density in units of $\mathrm{g} \mathrm{cm}^{-3}$; and $N_{0}$ is Avogadro's constant.

For the screened potential, the penetration factor is then given by

$$
\Gamma(E)=\exp \left(\frac{-2 r_{c}}{\hbar} \sqrt{2 \mu E} \int_{0}^{1}\left[\frac{1}{u} \exp (x(1-u))-1\right]^{1 / 2} d u\right),
$$

where $x=x(E)=r_{c} / r_{D}$. Here, $r_{c}$ is the classical turningpoint radius defined by $V_{\mathrm{sc}}\left(r_{c}\right)=E$. However, if $x$ is small, then $r_{c}$ for the screened potential is roughly that for the unscreened potential: $r_{c} \simeq Z_{1} Z_{2} e^{2} / E$. By expanding the exponential in the small- $x$ limit (to be justified below), we obtain

$$
\Gamma(E)=\exp [-2 \pi \eta(1-x / 2)]=e^{-2 \pi \eta} e^{x \pi \eta} .
$$

Although $x \pi \eta$ depends on the energy, the effect of the correction on the thermally-averaged cross section can be approximated by evaluating $x \pi \eta$ at the most probable energy of interaction,

$$
\begin{aligned}
E_{0} & =\left[\left(\pi \alpha Z_{1} Z_{2} k_{B} T\right)^{2}\left(m A c^{2} / 2\right)\right]^{1 / 3} \\
& =1.2204\left(Z_{1}^{2} Z_{2}^{2} A T_{6}^{2}\right)^{1 / 3} \mathrm{keV}
\end{aligned}
$$

where $m$ is the atomic mass unit, and $T_{6}$ is the temperature in units of $10^{6} \mathrm{~K}$. Then,

$$
\langle\sigma v\rangle \simeq \sqrt{\frac{8}{\pi \mu\left(k_{B} T\right)^{3}}} f_{0} \int_{0}^{\infty} d E S(E) \exp \left(-2 \pi \eta-E / k_{B} T\right),
$$

where the Salpeter factor $f_{0}$ is given by

$$
f_{0}=e^{x_{0} \pi \eta}=\exp \left(0.188 Z_{1} Z_{2} \zeta \rho^{1 / 2} T_{6}^{-3 / 2}\right),
$$

and

$$
x_{0}=x\left(E_{0}\right)=0.0133\left(Z_{1} Z_{2}\right)^{1 / 3} A^{-1 / 3} \rho^{1 / 2} T_{6}^{-7 / 6} \zeta .
$$

For the $p p$ reaction, $x_{0} \simeq 0.01$, which justifies the small- $x$ approximation used above. Equations (3)-(11) provide an alternative derivation of the Salpeter [1] weak-screening formula.

We now calculate numerically the cross section for the $p p$ reaction for a Coulomb potential and a screened Coulomb potential to compare with the WKB calculation of the screening correction. To do so, we note that the reaction rate is proportional to $\Lambda^{2}$ [7], where $\Lambda$ is the overlap integral of the proton-proton wave function and the deuteron wave function,

$$
\Lambda=\sqrt{\frac{a_{p}^{2} \gamma^{3}}{2}} \int u_{d}(r) u_{p p}(r) d r
$$

where $a_{p}$ is $p p$ scattering length, $\gamma=\sqrt{2 \mu E_{d}}$ is the deuteron binding wave number, and $E_{d}$ is the deuteron binding energy. The function $u_{d}(r)$ is the radial part of the $S$-state deuteron wave function. Our calculation here follows the approach and notation of Ref. [5].

For the purposes of this exercise, we use the McGee wave function [8] for the deuteron. If another wave function (which fits the deuteron data) is used, the overlap integral changes only slightly. Since we are here only investigating the effect of the screening correction to the reaction rate, our specific choice of the deuteron wave function is unimportant.

The radial wave function $u_{p p}(r)$ satisfies the radial Schrödinger equation,

$$
\frac{d^{2} u}{d r^{2}}-\left[\frac{1}{R r}+V_{\mathrm{nuc}}(r)\right] u=-k^{2} u
$$

where $R=\hbar^{2}\left(2 \mu e^{2}\right)^{-1}=28.8198 \mathrm{fm}, k=\mu v / \hbar$ is the center-of-mass momentum, and $V_{\text {nuc }}(r)$ is the short-range nuclear potential. For $V_{\text {nuc }}(r)$ we use an exponential potential which yields the observed value for the scattering length and effective range [5]. Again, the overlap integral turns out to be practically independent of the detailed shape of the nuclear potential (as long as it matches the measured scattering length and effective range), so the choice of nuclear potential is unimportant for determining the screening correction.

In the weak-screening case, Eq. (13) is replaced by

$$
\frac{d^{2} u}{d r^{2}}-\left[\frac{e^{-r / r_{D}}}{R r}+V_{\mathrm{nuc}}(r)\right] u=-k^{2} u \text {. }
$$


The solution to the Schrödinger equation is unique once the two boundary conditions are given. The first condition is $u(0)=0$. The other boundary condition is obtained by noting that the asymptotic behavior of the wave function for $r \gg r_{D}$ must be [9],

$$
u_{p p}^{\mathrm{Coul}}(r) \sim N_{\mathrm{Coul}} \sin \left(k r-\frac{1}{2 k R} \ln (2 k r)+\delta_{0}^{\mathrm{Coul}}\right),
$$

for the Coulomb potential, and

$$
u_{p p}^{\mathrm{sc}}(r) \sim N_{\mathrm{sc}} \sin \left(k r-\delta_{0}^{\mathrm{sc}}\right)
$$

for the screened potential, where $\delta^{\text {Coul }}$ and $\delta^{\text {sc }}$ are phase shifts. Fixing the incident flux of protons for the Coulomb and the screened-Coulomb interactions requires $N_{\text {Coul }}$ $=N_{\mathrm{sc}}$.

To solve this boundary-value problem, we integrate Eqs. (13) and (14) from $r=0$ with the condition $u(0)=0$ and $u^{\prime}(0)=1$ to a large distance (about $10 r_{D}$ ), and then test that the solutions converge to the form of Eqs. (15) and (16), respectively. From these numerical solutions, we obtain the normalizations $N_{\text {sc }}$ and $N_{\text {Coul }}$. We then use the calculated wave functions to evaluate the overlap integral in Eq. (12) both with and without screening. By squaring the ratio of the two overlap integrals, we determine numerically the screening correction to the cross section for the $p p$ reaction.

The difference between the WKB approximation and the numerical result for the $p p$ reaction in the Sun is negligible. For example, with the Debye-Huckel potential, the WKB and numerical screening corrections agree for $k=0.012$ $\mathrm{fm}^{-1}$ to better than $10^{-4}$. At even smaller values of $k$, the relative error is even smaller because the WKB approximation becomes increasingly valid as $\eta$ becomes larger [cf. Eq. (2)], which occurs as the center-of-mass energy becomes smaller [9]. We have also compared the WKB and numerical results for the alternative screening potential suggested by Dzitko et al. [2]. (For this potential, the WKB integral was evaluated numerically.) The WKB and Schrödinger results for the screening correction for this potential also agree to better than $10^{-4}$.

Our main result is that a numerical solution of the screened Schrödinger equation for the proton-proton reaction gives results in excellent agreement [to $\left.O\left(10^{-4}\right)\right]$ with the rate calculated analytically using the usual WKB approximation, as originally formulated by Salpeter. We have also shown that this agreement is independent of the precise details of the screening potential.

The cross sections for other fusion reactions in the $p p$ chain and $\mathrm{CNO}$ cycle are determined from extrapolation of laboratory measurements; they are not determined from first principles. Therefore, calculations analogous to those carried out here for the $p p$ reaction cannot be generalized straightforwardly to these other reactions. However, the WKB approximation becomes increasingly accurate as $\eta$ becomes larger. When evaluated at the energy of the Gamow peak, $\eta \propto\left(Z_{1}^{2} Z_{2}^{2} A\right)^{1 / 3}$, and this is smallest for the $p p$ reaction. Therefore, the WKB approximation should be even more accurate for the other fusion reactions in the $p p$ chain and CNO cycle.

A small correction. Here, we calculate a correction to Salpeter's screening formula and find it to be negligibly small. The integral in Eq. (9) is usually evaluated by expanding in a power series of the inverse of a large quantity $\tau$,

$$
\tau=3 E_{0} / k_{B} T=42.487\left(Z_{1}^{2} Z_{2}^{2} A T_{6}^{-1}\right)^{1 / 3} \text {. }
$$

The average product can then be written in a compact form [6]:

$$
\langle\sigma v\rangle=1.3005 \times 10^{-15}\left[\frac{Z_{1} Z_{2}}{A T_{6}^{2}}\right]^{1 / 3} f_{0} S_{\text {eff }} \exp (-\tau) \mathrm{cm}^{3} \mathrm{~s}^{-1}
$$

where $S_{\text {eff }}$ is expressed in keV barns. To first order in $\tau^{-1}$ [10],

$$
S_{\mathrm{eff}}=S\left(E_{0}\right)\left(1+\tau^{-1}\left[\frac{5}{12}+\frac{5 S^{\prime} E_{0}}{2 S}+\frac{S^{\prime \prime} E_{0}^{2}}{S}\right]_{E=E_{0}}\right) .
$$

Expressing the various quantities in terms of their values at $E=0$, we find $[10]$

$$
\begin{aligned}
S_{\text {eff }}\left(E_{0}\right) \simeq & S(0)\left[1+\frac{5}{12 \tau}+\frac{S^{\prime}\left(E_{0}+\frac{35}{36} k_{B} T\right)}{S}\right. \\
& \left.+\frac{S^{\prime \prime} E_{0}}{S}\left(\frac{E_{0}}{2}+\frac{89}{72} k_{B} T\right)\right]_{E=0} .
\end{aligned}
$$

More accurately, however, we should include the factor $e^{x \pi \eta}$ in the thermal-average integral, Eq. (7). To do so, we rewrite the integral as

$$
\begin{aligned}
\langle\sigma v\rangle= & \sqrt{\frac{8}{\pi \mu\left(k_{B} T\right)^{3}}} \int_{0}^{\infty} d E S(E) \\
& \times \exp \left(-2 \pi \eta-E / k_{B} T+x \pi \eta\right) .
\end{aligned}
$$

Introducing the dimensionless quantity $z=E / E_{0}$, the exponential can be written as

$$
-2 \pi \eta-E / k_{B} T+x \pi \eta=-\frac{2 \tau}{3} z^{-1 / 2}-\frac{\tau}{3} z+\frac{x_{0} \tau}{3} z^{-3 / 2}
$$

To first order in $x_{0}$, the minimum point of the exponent is thus shifted to $z=1-x_{0}$, or $E=E_{0}\left(1-x_{0}\right)$. Using Laplace's method [11] for asymptotic expansion of integrals, we find that the only $O\left(x_{0}\right)$ correction to Eq. (18) is in the expression for $S_{\text {eff }}$. To $O\left(\tau^{-1}, x_{0}\right), S_{\text {eff }}$ is obtained simply by replacing $E_{0}$ by $E_{0}(1-x)$ in Eq. (19). Expressed as $S(0)$, we have 


$$
\begin{aligned}
S_{\mathrm{eff}} \simeq S(0)\left[1+\frac{5}{12 \tau}+\frac{S^{\prime}(0)\left(E_{0}(1-x)+\frac{35}{36} k_{B} T\right)}{S(0)}\right. & \\
& \left.+\frac{S^{\prime \prime}(0) E_{0}}{S(0)}\left(\frac{E_{0}}{2}(1-2 x)+\frac{89}{72} k_{B} T\right)\right],
\end{aligned}
$$

where we have neglected terms of order $O\left(x_{0} / \tau\right)$. We see that there is an $O(x)$ correction to the $S^{\prime}$ and $S^{\prime \prime}$ terms. Since $x$ is small $\left(\sim 10^{-2}\right.$ for the $p p$ reaction at the core of the Sun), and the $S^{\prime}$ and $S^{\prime \prime}$ terms are generally small compared with the lowest-order term, these corrections are very small, typically $\sim 0.1 \%$. Therefore, the standard multiplicative correction factor $\left(f_{0}\right)$ should give a screened interaction rate which is accurate to $O(1 \%)$ in the weak-screening regime. Furthermore, since $x_{0}$ increases only very slowly with increasing mass number, the standard correction should also be accurate for other fusion reactions which are in the weakscreening regime.

This work was supported by U.S. DOE Contract No. DEFG02-92-ER 40699, NASA NAG5-3091, and the Alfred P. Sloan Foundation at Columbia. J.N.B. was supported at the Institute for Advanced Study by NSF Grant No. PHY9513835. M.K. thanks J. Applegate for useful conversations and J.N.B. thanks A. Gruzinov for valuable discussions. This work was initiated in response to a conjecture by A. Dar that the standard treatment of screening could cause an error of order $5 \%$ in the proton-proton reaction rate in the sun. We are grateful to A. Dar for this stimulating discussion.
[1] E. E. Salpeter, Aust. J. Phys. 7, 373 (1954).

[2] H. Dzitko et al., Astrophys. J. 447, 428 (1995).

[3] H. E. Mitler, Astrophys. J. 212, 513 (1977); C. Cararro, A. Schäfer, and S. E. Koonin, ibid. 331, 565 (1988); K. Langanke, in Solar Modeling, edited by A. B. Balantekin and J. N. Bahcall (World Scientific, Singapore, 1995); N. Shaviv and G. Shaviv, Astrophys. J. 468, 433 (1996); A. Dar and G. Shaviv, ibid. 468, 933 (1996).

[4] A. Gruzinov and J. N. Bahcall, Astrophys. J. (in press), astro-ph/9801028; L. S. Brown and R. F. Sawyer, Rev. Mod. Phys. 69, 411 (1997); A. V. Gruzinov, Astrophys. J. (in press), astro-ph/9702064; C. W. Johnson et al., Astrophys. J. 392, 320 (1992).
[5] M. Kamionkowski and J. N. Bahcall, Astrophys. J. 420, 884 (1994).

[6] J. N. Bahcall, Astrophys. J. 143, 259 (1966); see also J. N. Bahcall, Neutrino Astrophysics (Cambridge University Press, Cambridge, 1989).

[7] J. N. Bahcall et al., Rev. Mod. Phys. 54, 767 (1982).

[8] I. J. McGee, Phys. Rev. 151, 772 (1966).

[9] See e.g., L. D. Landau and E. M. Lifshitz, Quantum Mechanics, Non-Relativistic Theory (Pergamon Press, Oxford, 1977).

[10] J. N. Bahcall, Nucl. Phys. 75, 10 (1966).

[11] See e.g., C. M. Bender and S. A. Orszag, Advanced Mathematical Methods for Scientists and Engineers (McGraw-Hill, New York, 1978). 